\section{BCI Meeting 2005-Workshop on BCI Signal Processing: Feature Extraction and Translation}

Dennis J. McFarland, Charles W. Anderson, Klaus-Robert Müller, Alois Schlögl, and Dean J. Krusienski

\begin{abstract}
This paper describes the outcome of discussions held during the Third International BCI Meeting at a workshop charged with reviewing and evaluating the current state of and issues relevant to brain-computer interface (BCI) feature extraction and translation. The issues discussed include a taxonomy of methods and applications, time-frequency spatial analysis, optimization schemes, the role of insight in analysis, adaptation, and methods for quantifying BCI feedback.
\end{abstract}

Index Terms-Brain-computer interface (BCI), prediction, signal processing.

\section{INTRODUCTION}

Signal processing (i.e., feature extraction and translation) is a fundamental requirement for operation of brain-computer interfaces (BCIs), which take signals produced by the brain, and translates them into useful output commands with no intervention of muscles. One workshop held at the Third International BCI Meeting was charged with reviewing and evaluating the current state of BCI-related technology with regard to signal processing. The discussion covered six main issues: a taxonomy of methods and applications; time-frequency-spatial analysis; optimization schemes; the role of insight in analysis; adaptation; and methods for quantifying BCI feedback. The conclusions of these discussions are described below.

\section{TAXONOMY OF FEATURE EXTRACTION AND TRANSLATION METHODS}

The participants in the workshop provided summaries of the feature extraction and translation methods that they have used in their BCI research and development programs. The methods described in the responses were then used to develop categories that were arranged in a taxonomy of methods. The goals of this effort were: to discover how each laboratory's work relates to the collective effort of the BCI community; to prompt a discussion of which methods appear to be most

Manuscript received August 4, 2005. The National Institutes of Health (i.e., The National Institute of Biomedical Imagining and Biomedical Engineering, the National Center for Medical Rehabilitation Research of the National Institute of Child Health and Human Development, the National Institute on Deafness and Other Communication Disorders, and the Office of Rare Diseases) provided major funding for this meeting (R13EB00511401). Additional funding for the participation of students and Postdoctoral Fellows was provided by: The National Science Foundation; the Department of Defense Advanced Research Project Agency (DARPA); the Wadsworth Center (New York State Department of Health); Honeywell International; Cyberkenetics Neurotechnology Systems, Inc.; the Alfred E. Mann Foundation; g.tec Guger Technologies OEG; Cortech Solutions LLC; and Cleveland Medical Devices, Inc.

D. J. McFarland and D. J. Krusienski are with the Wadsworth Laboratories, New York State Department of Health, Albany, NY 12201 USA (e-mail: mcfarlan@wadsworth.org; dkrusien@wadsworth.org).

C. W. Anderson is with Colorado State University, Fort Collins, CO 80523 USA (e-mail: anderson@cs.colostate.edu).

K. R. Müller is with is with Fraunhofer FIRST (IDA), D-12489 Berlin, Germany (e-mail: klaus@ first.fhg.de).

A. Schlögl is with the Institute of Human-Computer Interfaces, University of Technology Graz, A-8010 Graz, Austria (e-mail: alois.schloegl@tugraz.at).

Digital Object Identifier 10.1109/TNSRE.2006.875637 fruitful for various applications; and to highlight new methods yet to be tried. The resulting taxonomy is outlined below. (It can also be found online ${ }^{1}$ along with summaries of the responses received from the workshop participants. The responses and the taxonomy make up a dynamic document that is updated as new responses and suggestions for taxonomy changes are received. Instructions online ${ }^{1}$ describe how to contribute.) The included references are by no means exhaustive, and major contributions have been left out of the listing. For example, not mentioned in the taxonomy are methods of feature selection and ways of combining multiple feature extraction and translation methods.

Taxonomy of Methods

Feature extraction

1) Time
a) Fourier transform [1]-[7]
b) Wavelets [8]
c) Autoregressive models [2], [9]-[12]
d) Bandpass filtering [13]
e) Template matching [14]
f) Kalman filter [11], [12], [14]
g) Spike detection [15]

2) Space
a) Laplacian filter [3], [9], [10], [16]-[18]
b) Principal components analysis [8], [14]
c) Independent components analysis [2], [16], [17]
d) Common spatial patterns [7], [13], [19]
e) Amplitudes [20]
f) Ratios and differences

3) Time-Space

a) Components analysis in time and space [21]

b) Multivariate autoregressive models [22]

c) Coherence [7]

4) Inverse models
a) Electroencephalogram (EEG) to electrocochleography $(\mathrm{ECoG})$
b) EEG to source dipoles [16], [17]

Feature translation

1) Linear
a) Linear discriminant analysis [7], [11], [12], [19], [21]
b) Perceptron [13]
c) Regression [3], [9], [10], [14], [15], regularized [2], [20], adaptive [18]

2) Nonlinear

a) Fixed structure

i) Quadratic discriminant analysis [11], [12]

b) Modifiable structure

i) Memory-based
A) k-nearest-neighbors [8], [21]
B) Support vector machines [2], [4], [6]
C) Partial least squares [6]

ii) Combinations of simple nonlinearities
A) Neural networks [23]
B) Decision trees [21]
C) Learning vector quantization [5]

iii) Generative models
A) Mixture of Gaussians [1]
B) Hidden Markov models

${ }^{1}$ http://www.cs.colostate.edu/eeg/taxonomy.html 
In addition, Mason, Bashashati, Fatourechi, and Birch are currently engaged in a related analysis of methods through an extensive survey of the literature. (It is available online. ${ }^{2}$ While the workshop's taxonomy outlined above focuses specifically on feature extraction and translation, the work of the latter investigators encompasses all aspects of BCI research and its applications.)

\section{TIME-FreQuency SPATIAL ANALYSIS}

BCI signal processing can be accomplished in stages. For example, the method currently implemented in BCI2000 for mu rhythm training consists of spatial filtering followed by spectral analysis [24]. However, it is also possible to consider these signal dimensions concurrently. For example, time-frequency spatial patterns can be used as features to predict electroencephalogram (EEG) recorded during right or left hand movement imagery [3]. Likewise, Anderson et al. [22] compared features extracted from EEG with univariate and multivariate autoregressive (AR) models for classifying mental tasks. In addition to the information contained in single channels, multivariate models also consider the time-dependent covariance between channels. Multivariate AR coefficients perform slightly better than those produced by univariate models.

Although the vast majority of BCI studies have treated these dimensions independently, and this is the simpler approach, such examples illustrate that it is possible to model the interdependencies between the time, frequency, and spatial dimensions of the signal. It depends, however, on the application, whether or not considering the interdependencies between these signal dimensions will provide a substantial improvement in performance.

\section{OPTIMIZATION SCHEMES}

Several aspects of a BCI can benefit from effective optimization including modeling, feature extraction, classifier training, and online adaptation. Approaches to optimization can be generally categorized into local techniques and global techniques [25]. Local techniques such as variants on gradient ascent/descent are well established, provably convergent, and can be effective for linear and simple nonlinear systems. However, these methods can grow increasingly complex for nonlinear systems such as neural and independent component analysis (ICA) networks and may become trapped in a local minimum. On the other hand, global techniques, such as evolutionary algorithms, are capable of avoiding local minima by intelligently searching the parameter space. Global methods should be considered in offline contexts unless simple local methods are sufficient or there are significant computational constraints. However, a main drawback of global techniques is that they require additional computational resources.

It is essential that an appropriate optimization technique is identified for a particular problem, and that the optimization technique not be a limiting factor in system design.

\section{INSIGHT From ALGORITHMS}

Algorithms used for feature extraction or classification should optimize BCI performance. In addition, it is desirable that these algorithms allow insight into and interpretation of the processes that are used by subjects to solve the BCI task they are confronted with. Insight and interpretation are desirable for two reasons. First, it is possible that users could employ various artifacts to solve the BCI task. In addition, many of the EEG features used in $\mathrm{BCI}$ research have an extensive literature that can be exploited and added to.

EEG features can be extracted with autoregressive modeling of the time series. The coefficients of the autoregressive model may serve as features or they may be used to estimate the power spectrum [26]. Use

\footnotetext{
${ }^{2}$ http://www.braininterface.org
}

of the power spectrum allows identification of known phenomena such as the mu rhythm or electromyography (EMG) contamination. In contrast, use of the coefficients as features does not allow for a straightforward interpretation. EEG features may be classified by means of linear algorithms, in which case it is possible to evaluate the relative weight of each individual feature. Nonlinear kernel-based methods could in principle assess such individual contributions in some high-dimensional feature space, but when projected back to the original input space, single-feature space dimensions typically correspond to an uninterpretable nonlinear mix of the input variables [27].

Linear methods that employ feature selection provide a possible solution. Common sequential methods that use dimensionality reduction, such as principal components analysis (PCA), do not guarantee good classification since the best discriminating component may not be among the largest principal components. An ideal strategy is to simultaneously construct a good classifier and select features for further explanation. Mathematical programming machines, such as the sparse Fisher's discriminant (SFD), can be used for this purpose. For example, Blankertz et al. [28] applied SFD to EEG measurements from a selfpaced finger tapping experiment. The SFD algorithm selected 68 out of 405 input dimensions that allowed left-right classification with good generalization. The selected input dimensions coincided well with what is expected from neurophysiology (i.e., high loadings for electrodes over sensorimotor areas). Thus state-of-the-art learning machines not only enable high classification accuracy, but also selection of a few features that match neurophysiological expectations.

Even linear methods may provide problems in interpretation when the predictors are correlated. For example, in a subject using a P300based matrix speller [24], a linear prediction of the target versus foil based on the 15 -sweep average of samples from $\mathrm{Cz}$ at 0 and $240 \mathrm{~ms}$ produced $r^{2}$ values of 0.004 and 0.073 , respectively, for the two features with univariate models, and 0.559 for the two in a bivariate model. With the bivariate linear regression model, feature weights were -0.155 for time 0 and 0.171 for $240 \mathrm{~ms}$. It is unlikely that the stimulus-related information contained in the EEG is nearly equivalent at 0 and $240 \mathrm{~ms}$. Rather it is probable that the feature at time 0 serves as a means of noise cancellation (i.e., it provides a baseline correction) and thus represents a suppressor variable [29]. Thus, weights from a multivariate model do not always represent the extent to which a feature provides independent predictive information.

\section{ADAPTATION}

Each step in the processes of feature extraction and translation may involve estimating parameters that are derived from the observed data. When the data are not stationary, some form of adaptation may optimize BCI performance. Successful adaptation should be both fast and stable. These properties are related, in part, to the selection of the appropriate time constant for adaptation. It is also important to consider the appropriate elements for adaptation. For example, it is possible to adapt the parameters of several signal processing stages separately. Alternatively, adaptation may involve the parameters of a single global feature space. At present, these issues remain largely unexplored.

It is important to appreciate that BCI systems involve feedback to the user in real time [26]. As a result, the user and the BCI represent two coupled adaptive systems. This may result in complex dynamics.

\section{CRITERIA FOR BCI EVALUATION}

There are a number of different measures available for evaluation of BCI systems. Perhaps the simplest of these is the error rate or classification accuracy. However, in order to calculate an error rate, a threshold must be applied. Alternatives that do not require specification of a threshold include signal-to-noise ratio, Cohen's Kappa [30], and $r^{2}$ [31]. The receiver-operator characteristics (ROC) curve, based 
on signal detection theory [32] provides a powerful tool for evaluating two-class problems.

Comparisons of accuracy or measures of association may be difficult when tasks vary in terms of parameters such as the number of alternatives that users can select or the rate at which selection occurs. Wolpaw et al. [26] suggested the use of bit-rate, based on Shannon's communication theory. Other measures based on Shannon's theory such as mutual information [33] are also available.

BCI performance can also be evaluated in terms of time to complete a particular task. For example, the number of letters selected per minute can be used to evaluate spelling devices based on different methodologies [34]-[36].

There is probably no single measure that is ideal for all applications. For example, simulations show that a $6 \times 6$ P300-based spelling matrix transmits information when the probability of a correct selection is below 0.5 . However, in copy spelling, if correction of errors is required, then reasonable performance in a time-to-complete measure is attained only with probabilities in excess of 0.7 .

\section{SUMMARY}

BCI research is a relatively new field and many alternative methods and concepts relevant to signal processing and feature extraction and translation need to be explored. Although many possible solutions have been proposed and demonstrated to work, it is necessary to compare these in well-designed systematic studies and on established data sets like the BCI Competition III [37].

\section{REFERENCES}

[1] J. del R. Millán, F. Renkens, J. Mourino, and W. Gerstner, "Brainactuated interaction," Artif. Intel., vol. 159, no. 1-2, pp. 241-259, Nov. 2004.

[2] P. Hammon, private communication, 2005.

[3] S. Lemm, B. Blankertz, G. Curio, and K.-R. Müller, "Spatio-spectral filters for improved classification of single trial EEG," IEEE Trans. Biomed. Eng., vol. 52, no. 9, pp. 1541-1548, Sep. 2005.

[4] R. Kaidar, private communication, 2005.

[5] G. Pfurtscheller, C. Neuper, D. Flotzinger, and M. Pregenzer, "EEG-based discrimination between imagination of right and left hand movement," Electroencephalogr. Clin. Neurophysiol., vol. 103, pp. 642-651, 1997.

[6] T. Rosipal, L. Trejo, and B. Matthews, "Kernel PLS-SVC for linear and nonlinear classification," in Proc. 20th Int. Conf. Machine Learning (ICML-2003), Washington, DC, 2003, pp. 640-647.

[7] C. Brunner, personal communication, 2005.

[8] M. Fatourechi, A. Bashashati, R. Ward, and G. Birch, "A hybrid genetic algorithm approach for improving the performance of the LF-ASD brain computer interface," in Proc. Int. Conf. Acoust. Speech Signal Process., 2004, vol. 5, pp. 345-348.

[9] J. R. Wolpaw and D. J. McFarland, "Control of a two-dimensional movement signal by a non-invasive brain-computer interface in humans," Proc. Nat. Acad. Sci., vol. 101, pp. 17849-17854, 2004.

[10] D. J. McFarland and J. R. Wolpaw, "Sensorimotor rhythm-based braincomputer interface (BCI): Feature selection by regression improves performance," IEEE Trans. Neural Syst. Rehabil. Eng., vol. 13, no. 3, pp. 372-379, Sep. 2005.

[11] C. Neuper, A. Schlogl, and G. Pfurtscheller, "Enhancement of left-right sensorimotor EEG differences during feedback-regulated motor imagery," J. Clin. Neurophysiol., vol. 16, no. 4, pp. 373-382, Jul. 1999.

[12] A. Schlögl, K. Lugger, and G. Pfurtscheller, "Using adaptive autoregressive parameters for a brain-computer-interface experiment," in Proc. 19th Annu. Int. Conf. IEEE Eng. Med. Biol. Soc., 1997, vol. 19, pp. 1533-1535.

[13] Y. Li, X. Gao, and S. Gao, "Classification of single-trial electroencephalogram during finger movement," IEEE Trans. Biomed. Eng., vol. 51, no. 6, pp. 1019-1025, Jun. 2004.

[14] G. Gage, K. Ludwig, K. Otto, E. Ionides, and D. Kipke, "Naive coadaptive cortical control," J. Neural Eng., vol. 2, pp. 52-63, 2005.
[15] R. Stein, D. Weber, Y. Aoyagi, A. Prochazka, J. Wagenaar, S. Shoham, and R. Normann, "Coding of position by simultaneously recorded sensory neurons in the cat dorsal root ganglion," J. Physiol., vol. 560, pp. 883-896, 2004.

[16] B. Kamousi, Z. Liu, and B. He, "Classification of motor imagery tasks for brain-computer interface applications by means of two equivalent dipoles analysis," IEEE Trans. Neural Syst. Rehabil. Eng., vol. 13, no. 2, pp. 166-171, Jun. 2005.

[17] L. Qin, L. Ding, and B. He, "Motor imagery classification by means of source analysis for brain computer interface applications," J. Neural Eng., vol. 1, pp. 135-141, 2004.

[18] D. Krusienski, G. Schalk, D. McFarland, and J. Wolpaw, "Tracking of the mu rhythm using an empirically derived matched filter," Proc. IEEE Int. Conf. Neural Eng., vol. , pp. 86-89, Mar. 2005.

[19] G. Dornhege, B. Blankertz, G. Curio, and K. Müller, "Boosting bit rates in non-invasive EEG single-trial classifications by feature combination and multi-class paradigms," IEEE Trans. Biomed. Eng., vol. 51, no. 6, pp. 993-1002, Jun. 2004

[20] B. Blankertz, G. Dornhege, C. Schaefer, R. Krepki, J. Kohlmorgen, K. Mueller, V. Kunzmann, F. Losch, and G. Curio, "Boosting bit rates and error detection for the classification of fast-paced motor commands based on single-trial EEG analysis," IEEE Trans. Neural Syst. Rehabil. Eng., vol. 11, no. 2, pp. 127-131, Jun. 2003.

[21] M. Kirby and C. Anderson, "“'Geometric analysis for the characterization of nonstationary time-series,"," in Springer Applied Mathematical Sciences Series Celebratory Volume for the Occasion of the 70th Birthday of, L. Sirovich, E. Kaplan, J. Marsden, and K. R. K. Sreenivasan, Eds. New York: Springer-Verlag, 2003, ch. 8, pp. 263-292.

[22] C. W. Anderson, E. A. Stolz, and S. Shamsunder, "Multivariate autoregressive models for classification of spontaneous electroencephalogram during mental tasks," IEEE Trans. Biomed. Eng., vol. 45, no. 3, pp. 277-286, Mar. 1998.

[23] C. W. Anderson and Z. SijerŸ ci' c, "Classification of EEG signals from four subjects during five mental tasks," in Solving Engineering Problems with Neural Networks: Proceedings of the Conference on Engineering Applications in Neural Networks (EANN'96), A. B. Bulsari, S. Kallio, and D. Tsaptsinos, Eds., Turkey, 1996, pp. 407-414.

[24] G. Schalk, D. J. McFarland, T. Hinterberger, N. Birbaumer, and J. R. Wolpaw, "BCI2000: A general-purpose brain-computer interface (BCI) system," IEEE Trans. Biomed. Eng., vol. 51, no. 6, pp. 1034-1043, Jun. 2004

[25] D. J. Krusienski and W. K. Jenkins, "Design and performance of adaptive systems based on structured stochastic optimization strategies," IEEE Circuits Syst. Mag., vol. 5, no. 1, pp. 8-20, 2005.

[26] J. R. Wolpaw, N. Birbaumer, D. J. McFarland, G. Pfurtscheller, and T. M. Vaughan, "Brain-computer interfaces for communication and control," Clin. Neurophysiol., vol. 113, pp. 767-791, 2002.

[27] B. Scholkopf, S. Mika, C. J. C Burges, P. Knirsch, K. R. Muller G. Ratsch, and A. J. Smola, "Input space versus feature space in kernal-based methods," IEEE Trans. Neural Netw., vol. 10, no. 5, pp. 1000-1017, Sep. 1999.

[28] B. Blankertz, G. Curio, and K.-R. Müller, Classifying single trial EEG: Towards brain computer interfacing, T. G. Diettrich, S. Becker, and Z. Ghahramani, Eds. Cambridge, MA: MIT Press, 2002, vol. 14, Advances in Neural Information Processing Systems, pp. 157-164.

[29] L. Friedman and M. Wall, "Graphical views of supression and multicollinearity in multiple linear regression," Amer. Statistician, vol. 59, pp. 127-136, 2005.

[30] A. Schlogl, F. Lee, H. Bishof, G. Pfurtscheller, and G. , "Characterization of four-class motor imagery data for the BCI-competition 2005"," J. Neural Eng., vol. 2, p. 14, 2005.

[31] H. Sheikh, D. J. McFarland, W. A. Sarnacki, and J .R. Wolpaw, "EEGbased communication: Characterizing EEG control and performance relationship," Neurosci. Lett., vol. 345, pp. 89-92, 2003.

[32] M. D. Green and J. A. Swets, Signal detection theory and psychophysics. Huntington, NY: Krieger, 1966.

[33] A. Schlogl, C. Neuper, and G. Pfurtscheller, "Estimating the mutual information of an EEG-based brain-computer interface," Biomedizinische Technik, vol. 47, pp. 3-8, 2002.

[34] N. Birbaumer, N. Ghanayim, T. Hinterberger, I. Iversen, B Kotchoubey, A. Kubler, J. Perelmouter, E. Taub, and H. Flour, "A spelling device for the paralysed," Nature, vol. 398, pp. 297-298, 1999.

[35] E. Donchin, K. M. Spencer, and R. Wijesinghe, "The mental prosthesis: Assessing the speed of a P300-based brain-computer interface," IEEE Trans. Rehabil. Eng., vol. 8, no. 2, pp. 174-179, Jun. 2000. 
[36] R. Scherer, G. R. Muller, C. Neuper, B. Graimann, and G. Pfurtscheller, "An asynchronious controlled EEG-based virtual keyboard: Improvement of the spelling rate," IEEE Trans. Biomed. Eng., vol. 51, no. 6, pp. 979-984, Jun. 2003.

[37] B. Blankertz, K.-R. Müller, D. Krusienski, J. R. Wolpaw, A. Schlögl, G. Pfurtscheller, J. del R. Millán, M. Schröder, and N. Birbaumer, "The BCI competition III: Validating alternative approachs to actual BCI problems," IEEE IEEE Trans. Neural Syst. Rehabil. Eng., vol. 51, no. 14, pp. 153-159, Jun. 2006.

\section{BCI Meeting 2005-Workshop on Signals and Recording Methods}

Jonathan R. Wolpaw, Gerald E. Loeb, Brendan Z. Allison, Emanuel Donchin, Omar Feix do Nascimento, William J. Heetderks, Femke Nijboer, William G. Shain, and James N. Turner

\begin{abstract}
This paper describes the highlights of presentations and discussions during the Third International BCI Meeting in a workshop that evaluated potential brain-computer interface (BCI) signals and currently available recording methods. It defined the main potential user populations and their needs, addressed the relative advantages and disadvantages of noninvasive and implanted (i.e., invasive) methodologies, considered ethical issues, and focused on the challenges involved in translating BCI systems from the laboratory to widespread clinical use. The workshop stressed the critical importance of developing useful applications that establish the practical value of BCI technology.
\end{abstract}

Index Terms-Brain-computer interface (BCI), electrophysiological signals, rehabilitation.

\section{INTRODUCTION}

This workshop, which was part of the 2005 BCI meeting, addressed the characteristics, advantages, and disadvantages for brain-computer interface (BCI) use of different brain signals and recording methods with particular reference to development of clinical applications. The major issues considered included the characteristics, capacities, and needs of people likely to benefit from a BCI; the advantages and disadvantages of electroencephalographic (EEG), electrocorticographic (ECoG), and intracortical signals; other possible signal modalities; the ethical issues associated with BCI research and use; and the transition from the laboratory to widespread clinical use. The participants

Manuscript received February 27, 2006; revised March 27, 2006. The National Institutes of Health (i.e., The National Institute of Biomedical Imagining and Biomedical Engineering, the National Center for Medical Rehabilitation Research of the National Institute of Child Health and Human Development, the National Institute on Deafness and Other Communication Disorders, and the Office of Rare Diseases) provided major funding for this meeting (R13EB00511401). Additional funding for the participation of students and postdoctoral fellows was provided by: The National Science Foundation; the Department of Defense Advanced Research Project Agency (DARPA); the Wadsworth Center (New York State Department of Health); Honeywell International; Cyberkenetics Neurotechnology Systems, Inc.; the Alfred E. Mann Foundation; g.tec Guger Technologies OEG; Cortech Solutions, LLC; and Cleveland Medical Devices, Inc.

The authors are with Wadsworth Center, Albany, NY 12201-0509 USA (e-mail: wolpaw@wadsworth.org; gloeb@usc.edu; ballison@scripps.edu; donchin@shell.cas.usf.edu; omar@smi.auc.dk; heetderw@mail.nih.gov; femke.nijboer@uni-tuebingen.de; shain@wadsworth.org; turner@wadsworth. org).

Digital Object Identifier 10.1109/TNSRE.2006.875583 included clinical and basic neuroscientists, cell biologists, engineers, biophysicists, psychologists, and clinicians. The format included individual presentations and panel-led discussions.

\section{USER POPULATIONS}

BCI users are often categorized according to the disorders responsible for their disabilities, such as amyotrophic lateral sclerosis (ALS), brainstem stroke, spinal cord injury, or cerebral palsy. However, decisions regarding whether or how BCI technology might be useful to these users usually depend more on the extent of their disability than on its origin. In this regard, potential users fall into three relatively distinct classes.

The first class consists of people who are truly totally locked-in (e.g., due to end-stage ALS or severe cerebral palsy), who have no remaining useful neuromuscular control of any sort, including no eye movement. Although this class is very small, it is often considered to be the first target group for BCI applications. However, in reality, efforts to demonstrate effective BCI operation by these individuals encounter a number of difficult challenges. It is often unclear, for example, whether cognitive functions remain intact, whether vision is adequate to support BCI operation, and if not, whether an auditory or other alternative will suffice, and whether the user can or will maintain a state of alertness adequate for reliable BCI operation. In practice to date, these or related issues have been major impediments to BCI usage by these users. Each person requires a comprehensive and individualized approach that goes far beyond the much simpler procedures effective in those who are not totally locked-in. On the other hand, if people progressing toward this level of disability (e.g., those in the early or middle stages of ALS) begin to use $\mathrm{BCI}$ technology before they become totally locked-in, they may be able to continue to use it effectively after they lose all motor function.

The second class of potential BCI users comprises those who retain a very limited capacity for neuromuscular control. This group includes people who retain some useful eye movement or enough limb muscle function to operate a single-switch system. Such control is often slow, unreliable, or easily fatigued [5]. This group is much larger than the first, and includes many people with late-stage ALS, brainstem stroke, and severe cerebral palsy. The advent of widely available life-support technology, particularly ventilators, enables these individuals to survive indefinitely, and numerous studies now show that with adequate physical and social support they can lead lives that they and their families and friends consider worthwhile and enjoyable [12], [20], [23], [27], [32], [45]. Thus, there is substantial impetus for developing BCI communication and control technology for this group. Furthermore, current or readily achievable BCI systems may provide communication and control capacities (e.g., for simple word processing, environmental control, entertainment access) comparable to, or even better than, that achievable with their residual neuromuscular control.

The third class of potential BCI users, which is the largest of all, includes those who still retain (and can be expected to continue to retain) substantial neuromuscular control, particularly speech and/or hand control, and can, therefore, operate a wide range of assistive communication and control devices. For this group, as well as for users without disabilities, BCI technology, whether currently available or likely to be available in the near future, has little to offer (though it might be useful in very specific situations, such as when hands-free control is required). It will only be if and when BCI speed, accuracy, and precision of control substantially exceed current levels that this technology will become a significant option for this class of potential users. 